\title{
Occurrence of Buffalo Hump and Laryngeal Cancer in a HIV-1 Infected Patient with Metabolic Disorders Receiving a Rescue HAART Regimen
}

\author{
Alessandra Fantauzzia , Massimo Fusconi ${ }^{b}$, Caterina Tripodi ${ }^{b}$, Cristina Bigellib, \\ Carmen Barbato ${ }^{a}$, Giancarlo Ceccarellic ${ }^{\text {, Ivano Mezzaroma }}{ }^{\mathrm{a}, \mathrm{d}}$
}

\begin{abstract}
Reported cases of laryngeal squamous cell carcinomas are rare in HIV-1 infected patients. We describe a 68 year-old heavy smoker HIV-1 positive male receiving a salvage combination antiretroviral therapy, who developed a differentiated laryngeal cancer staging T3N0Mx after a 21 year history of HIV-1 infection. The patient also presented a severe fat redistribution with "buffalo hump" accompanied by subcutaneous adipose tissue lipoatrophy at arms and legs, and a dis-metabolic syndrome. Head and neck movements were severely limited by this voluminous mass. The presence of immunesuppression at the diagnosis with a relatively low grade staging of the neoplasm determined the choice of a combined chemo-radiation therapy, taking also in account the high infective risk linked to the surgery approach compared to that of acute toxicity reactions. A two years disease free follow up reinforced the needs of an early and accurate screening for neoplasms in HIV-1 infected patients.
\end{abstract}

Keywords: HIV-1; HAART; Buffalo hump; HARS; Laryngeal cancer; Radiation therapy

\section{Introduction}

Highly active antiretroviral therapy (HAART) has dramati-

Manuscript accepted for publication February 7, 2012

aDepartment of Clinical Medicine, "Sapienza" - University of Rome,

Rome, Italy

${ }^{b}$ Department of ENT, Audiology and Phoniatrics,

"Sapienza" - University of Rome, Rome, Italy

cDepartment of Public Health and Infectious Diseases,

"Sapienza" - University of Rome, Rome, Italy

${ }^{\mathrm{d}}$ Corresponding author: Ivano Mezzaroma, Department of Clinical

Medicine, "Sapienza" - University of Rome, Viale dell’Universita 37, 00185 Rome, Italy. Email: ivano.mezzaroma@uniroma1.it

doi: $10.4021 / \mathrm{jmc} 569 \mathrm{w}$ cally changed prognosis and life expectancy of human immunodeficiency virus type-1 (HIV-1) infected patients. Opportunistic infections and other immunodeficiency-associated conditions are minimized, and patients can live longer. However, HAART can determine some quite debilitating side effects $[1,2]$ such as the HIV-1-associated adipose redistribution syndrome (HARS) [3]. Its primary characteristic is an increase of visceral adipose tissue (VAT), often accompanied by a lipoatrophy of the subcutaneous tissue in the face, arms, and legs [4]. In addition, adipose tissue may accumulate in pubis, neck or trunk $[5,6]$ and in dorso-cervical area (buffalo hump) [7, 8]. Etiopathogenesis of HARS is still unknown. Infection stage, host factors and HAART exposure are involved $[9,10]$. "Buffalo hump" is a common presentation of HARS and can cause significant disfigurement to the patient. A recent study estimated in $6 \%$ its prevalence among HAART-treated patients [11]. Buffalo hump may result from alterations of the adipocytes at those sites. In fact, these cells express the brown fat uncoupling protein-1 (UCP-1) gene, thus suggesting disturbances in the brown-versus-white adipocyte differentiation pattern [12]. Another hypothesis is the involvement of adipocyte mitochondrial disturbances [13] with a site-specific compensatory response to metabolic dysregulation, or an enhanced local toxicity elicited by HIV-1 itself and/or by HAART. Although medical treatments including recombinant growth hormone and anabolic steroids [14, 15] have been used with improvements, surgical approach remains the mainstay of correction of buffalo hump $[16,17]$.

HIV-1 infected individuals are at increased risk of developing non-AIDS cancers, particularly those associated with infections and smoking. Non-AIDS cancers were greater among men than women and among those with AIDS than those without AIDS; however, no substantial difference was observed by HAART era. Patients having a history of tobacco or alcohol abuse, should be carefully examined for head and neck tumors that are likely to be more aggressive [18]. In uninfected general population, laryngeal cancer accounts for $2 \%$ of all cancers and $60 \%$ of those of the head and neck and occurs most frequently in males [19]. In HIV-1 patients laryngeal cancer incidence is 1.5 times greater than in general population [20]. Clinical course of the tumor may be more aggressive and this may be related to an impairment of im- 

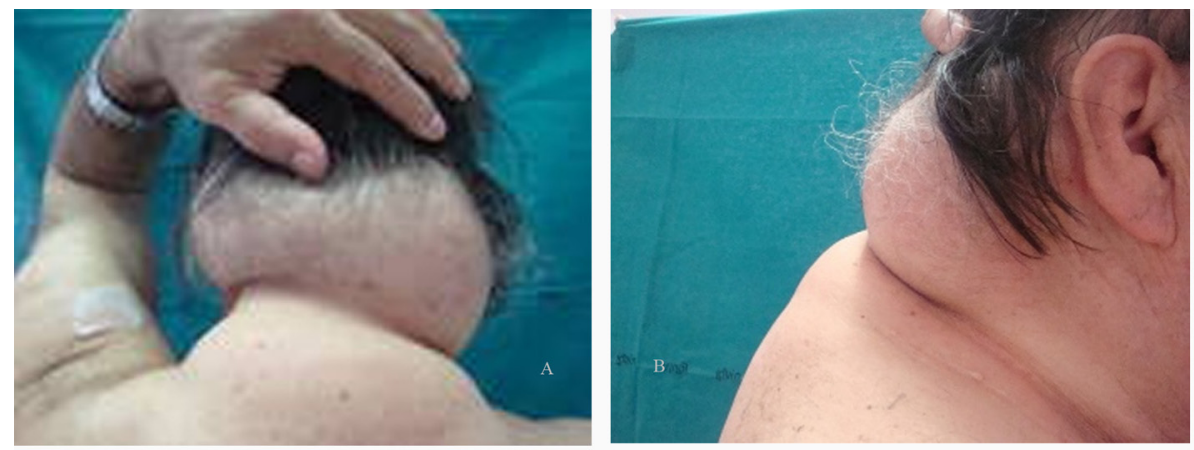

Figure 1. (A) dorsal and (B) lateral views of lipoaccumulation.

mune surveillance mechanisms secondary to HIV-1 [21, 22].

Treatment of laryngeal cancer involves many disciplines and requires more caution in HIV-1 patients.

We describe the outcome of a HIV-1 infected subject under rescue HAART, who developed a laryngeal cancer in the setting of a buffalo hump and metabolic disturbances. The relationship between the overlapping pathologies is analyzed.

\section{Case Report}

On March 1990, a 68 year-old heterosexual man presenting with severe thrombocytopenia and recurrent herpes zoster was diagnosed as having a HIV-1 infection stage B3, according to the Centers for Disease Control classification system [23]. A generalized lymphoadenopathy with CD4+ T cell count of 180 cells $/ \mu \mathrm{L}$ was present and a zidovudine-based antiretroviral therapy was started. Hepatotropic virus serology (HAV, HBV and HCV) was negative. Familiarity for hypertension and dyslipidemia, and tobacco smoking were also present. Up to 2000, he changed several antiretroviral treatments, from mono-dual therapies with nucleoside reverse transcriptase inhibitors (NRTIs) to protease inhibitor (PI)-based triple combination treatments (including indina- vir). A partial immune recovery was observed and plasma HIV-RNA levels sometimes reached undetectable values, with occasional high triglyceride and cholesterol levels. A fat redistribution was also evident, with VAT increase and lipoatrophy on arms and legs, and an initial buffalo hump. On January 2001, treatment was replaced with lopinavir/ ritonavir plus abacavir/lamivudine/zidovudine-fixed dose (inconsistent compliance and virological failure), with worsening in fat redistribution and lipid levels, needing lipid-lowering therapy. In November 2007, patient showed a severe immunodeficiency $(\mathrm{CD} 4+13 \%, 120$ cells $/ \mu \mathrm{L})$ with 56.000 copies $/ \mathrm{mL}$ of HIV-RNA so, based on the results of a genotypic resistance test, darunavir/ritonavir + raltegravir + tenofovir and lamivudine/zidovudine fixed-dose were started. Three months later, plasma HIV-RNA reached undetectable levels $(<50$ copies $/ \mathrm{mL})$ and $\mathrm{CD} 4+$ raised to $19 \%$ (202 cells $/ \mu \mathrm{L}$ ). Subsequently, hypertension and chronic atrial fibrillation was diagnosed and an anti-arrhythmic, anticoagulant and anti-platelet therapy added to HAART, but patient developed an ischemic stroke in the right anterior cerebral artery territory. In November 2008, after an acute bronco-pneumonia, he was treated for a left carotid stenosis $(85 \%)$ and developed a reactive depression with behavioral changes. On April 2009, HAART was simplified with darunavir/ritonavir plus raltegravir and emtricitabine/tenofovir

Table 1. Determinations of Serum Panel Cytokines

\begin{tabular}{lllcc}
\hline Cytokine & Pre-CHT & Post-CHT & Normal values* & Units \\
\hline Adiponectin & 7.38 & 8.12 & $0.87-21.4$ & $\mu \mathrm{g} / \mathrm{mL}$ \\
Leptin & 34.6 & 36.3 & $2.21-11.15$ & $\mathrm{ng} / \mathrm{mL}$ \\
Resistin & 12.05 & 11.53 & $8.2 \pm 3.0$ & $\mathrm{ng} / \mathrm{mL}$ \\
IL-6 & 32.9 & 36.0 & $0-10.09$ & $\mathrm{pg} / \mathrm{mL}$ \\
\hline
\end{tabular}

* in healthy males. 
fixed-dose. Viro-immunological assessment showed HIVRNA $<50$ copies $/ \mathrm{mL}, \mathrm{CD} 4+18 \%(295$ cells $/ \mu \mathrm{L})$, and normal metabolic parameters. On December 2009, triglycerides and cholesterol were uncontrolled despite the lipid-lowering treatment, whereas buffalo hump determined a functional limitation in head and neck movements. Moreover, patient referred recent onset of persistent cough and dysphonia with dysphagia. A fiberscope examination showed an exophytic neoformation with irregular limits (diameter $2 \mathrm{~cm}$ ) in the laryngeal epiglottis face. A CT angiography confirmed the neoformation $(2.2 \times 1.8 \mathrm{~cm})$ in the supraglottic region involving the laryngeal side of epiglottis (with infiltration of the pre-epiglottic and paraglottic fat) and extension to the right epiglottal ear pocket; no thyroid cartilage erosions or nodes involvement; severe lipodystrophy of the subcutaneous adipose tissue in the submandibular, retronucal and dorsal region (antero-posterior diameter $7.4 \mathrm{~cm}$, transverse 16 $\mathrm{cm}$, longitudinal $8.8 \mathrm{~cm}$.). The tumor staging was T3N0Mx. Histological examination showed an infiltrating squamous cell carcinoma. According to the 2008 American Cancer Society guidelines [24], patient underwent a radiation therapy (RT) and platinum-based chemotherapy (CHT) protocol, without HAART discontinuation. The treatment was well tolerated, with only a grade 2 dysphagia and mucositis. A CT scan showed the laryngeal cancer disappearance without significant changes in the buffalo hump mass (antero-posterior diameter $6.0 \mathrm{~cm}$, transverse $15 \mathrm{~cm}$, longitudinal $8.8 \mathrm{~cm}$ ), despite patient's 5 kilograms weight loss (Fig. 1). Immune activation and metabolic profiles were assessed by plasma concentrations of IL-6 (Enzo Life Sciences, Ann Arbor, MI, USA), leptin (Adipogen International, SOUTH KOREA), adiponectin (Enzo Life Sciences, Ann Arbor, MI, USA) and resistin (Adipogen International, SOUTH KOREA), using commercially available kits, being these cytokines a marker of altered lipid and glucose metabolism in HIV-1 patients receiving HAART.

Two measurements were performed: the first one before the diagnosis of laryngeal cancer and the other one during the second year of follow-up (Table 1).

All assays were performed in duplicate. Low adiponectin with high leptin and IL-6 levels were found before cancer diagnosis. At the second determination similar results were found. Patient's metabolic profile was evaluated by the homeostasis model assessment of insulin resistance (HOMA-IR), using the standardized formula (fasting glucose $(\mathrm{mmol} / \mathrm{L}) \times$ fasting insulin $(\mathrm{mU} / \mathrm{L}) / 22.5)$, and by the body mass index (BMI) calculation. HOMA-IR index was 0.24 , whereas BMI resulted 30.06 (slightly high). Patient then entered in a follow-up program with bimonthly clinical and fiberscope examination in the first year, quarterly in the second year, then every six months. He is currently followed on regular basis, and no signs of relapse are present; HIV RNA levels are always $<50$ copies $/ \mathrm{mL}$ and CD4+ T cell counts are increased from $89 / \mu \mathrm{L}(12 \%$, nadir post-CHT) to
$273 / \mu \mathrm{L}(18 \%)$

\section{Discussion}

Causes of metabolic alterations and adipose tissue modifications in HIV-1 patients during HAART are different and controversial, in fact antiretroviral drugs, hormonal changes and cytokine level alterations are involved. Moreover it has been hypothesized that adipocyte functions may play an important role in the development of HARS-associated metabolic abnormalities. Adipocytes are metabolically and hormonally active, secreting proteins such as plasminogen activator inhibitor type 1, TNF- $\alpha$, IL-6, and leptin [25-26]. Some antiretroviral drugs inhibit differentiation and induce insulin resistance and apoptosis in adipose cells, both in vitro and in vivo. In vitro, PIs and NRTIs increase secretion of pro-inflammatory cytokines such as TNF- $\alpha$, IL- 6 and IL-1 $\beta$, which are involved in altered adipocyte functions, and decrease production of adiponectin, a positive modulator of insulin sensitivity. Adipocytes also produce a protein (resistin) that plays an important function as a link between obesity and insulin resistance. Adiponectin is a structural homologue of the TNF- $\alpha$ family of trimeric cytokines with anti-inflammatory properties that antagonize those of TNF- $\alpha$ [27]. These disorders probably result in an increased release of free fatty acids by insulin-resistant adipose tissue. In fact, similar alterations are observed in fat and serum from HIV-1-infected lipodystrophic patients under HAART [28].

A cytokine production alteration plays a pivotal role in the development of insulin resistance, endothelial dysfunction, and abnormal fibrinolysis, those are frequently described in metabolic syndrome. Hyper-insulinemia, type-2 diabetes, hypertension, hyper-lipidemia and coronary heart disease (CHD) also characterize this syndrome, often occurring in HIV-1 patients. Moreover, adiponectin negatively correlated with BMI, whereas its reduced levels have been associated with insulin resistance, high cholesterol and hyper-triglyceridemia and these alterations are involved in increased immune activation, responsible of the progressive immune depletion and, possibly, of the increased risk of malignancies. In fact, our patient showed impaired cholesterol and triglyceride metabolism, reduced adiponectin levels and high pro-inflammatory cytokine production, as described in HIV-1 lipodystrophyc patients. Moreover, HOMA index and BMI value were slightly high, as described in patients with mild obesity. These findings strongly suggest the need to monitor patient for early detection of type-2 diabetes. RT/ CHT protocol showed no impact on metabolic abnormalities, as well as on cytokine levels, that remained unchanged during follow up.

The risk of non-AIDS cancers among HIV-1 infected patients is much higher than in uninfected population, even after adjusting for age, gender, and race. Potential causes of 
this trend could be related to differences in lifestyle and rates of traditional cancer risk factors among HIV-1 patients, as well as to other viral infections (or HIV-1 itself), immune deficiency and immune activation [26].

In HIV-1 infected patients RT, and especially CHT, may cause life-threatening complications such as acute toxicity reactions [29]. Subjects with a good performance status can be safety treated with standard radical RT if the CD4+ T cell count is $>200$ cells $/ \mu \mathrm{L}$ [30]. Occasional reports of laryngeal cancer presenting a response to RT in HIV-1 patients with severe immune-suppression are described [31]. Following surgery, AIDS patients may have worse wound healing and a greater tendency to contract infections. In this case, a combined RT/CHT approach was chosen taking in account the neoplasm staging and the presence of severe immune suppression at the diagnosis, not allowing patient to tolerate a surgical treatment. Furthermore, concurrent RT plus cisplatin is the standard organ-preservation protocol in patients with locally advanced laryngeal cancer [32]. Neck and buffalo hump adipose tissues did not hamper an effective RT response of the laryngeal cancer. Furthermore, the fatty mass volume was not affected by RT, as showed by the CT-scans and described for normal adipose tissue. Even if in previous reports buffalo hump was assimilated to a tissue with a high proliferation activity [33], our observations indicate that in this context adipocytes have a metabolic profile similar to those present in other sites.

The peculiarity of the case described lies in the relatively rare occurrence in a HIV-1 infected subject of a laryngeal cancer with long disease-free survival after RT/CHT, the concurrent buffalo hump with metabolic syndrome, and the lack of opportunistic complications despite iatrogenic worsening of immunodeficiency. In the past decade we observed only 4 cases of laryngeal cancer, including the present one, in a cohort of 800 subjects with HIV-1 disease. All were presenting an advanced TNM staging and died within 6 - 12 months from the diagnosis. The not aggressive trend observed in this subject, despite several concomitant pathologies, may be probably related to TNM staging (T3N0Mx) and early diagnosis. Furthermore, presence of a high performance score and lack of severe immunodeficiency before RT/CHT, with a low grade toxicity observed, and the protective role exerted by HAART could all have contributed to the favourable outcome.

Since it has been hypothesized that in HIV-1 infected patients lipodystrophy would be a marker of early metabolic syndrome, a correct management of HIV-1 infected patients need to individualize antiretroviral therapy, choosing drugs with lower metabolic impact, as recommended in current guidelines [34]. Furthermore, considering the HAART-related life expectancy increase and the presence of lifestyle-related risk factors, it is important in HIV-1 infected patients to implement an expanded and accurate screening for an early detection and treatment of cancers.

\section{Conflict of Interest}

All authors declare that they have no conflict of interest.

\section{References}

1. Grinspoon S, Carr A. Cardiovascular risk and body-fat abnormalities in HIV-infected adults. N Engl J Med. 2005;352(1):48-62.

2. Wohl DA, McComsey G, Tebas P, Brown TT, Glesby MJ, Reeds D, Shikuma C, et al. Current concepts in the diagnosis and management of metabolic complications of HIV infection and its therapy. Clin Infect Dis. 2006;43(5):645-653.

3. Lichtenstein K, Balasubramanyam A, Sekhar R, Freedland E. HIV-associated adipose redistribution syndrome (HARS): definition, epidemiology and clinical impact. AIDS Res Ther. 2007;4:16.

4. Carr A. HIV lipodystrophy: risk factors, pathogenesis, diagnosis and management. AIDS. 2003;17 Suppl 1:S141-148.

5. Palella FJ, Jr., Chmiel JS, Riddler SA, Calhoun B, Dobs A, Visscher B, Kingsley L. A novel pattern of lipoaccumulation in HIV-infected men. JAMA. 2006;296(7):766768.

6. Guaraldi G, Orlando G, Squillace N, Roverato A, De Fazio D, Vandelli M, Nardini G, et al. Prevalence of and risk factors for pubic lipoma development in HIV-infected persons. J Acquir Immune Defic Syndr. 2007;45(1):72-76.

7. Lo JC, Mulligan K, Tai VW, Algren H, Schambelan M. "Buffalo hump" in men with HIV-1 infection. Lancet. 1998;351(9106):867-870.

8. Saint-Marc T, Touraine JL. "Buffalo hump" in HIV-1 infection. Lancet. 1998;352(9124):319-320.

9. Lauenroth-Mai E, Schlote F. HIV-associated lipodystrophy syndrome: LioN-HAART cohort: (lipodystrophy in patients on nucleoside-based HAART). Highly active antiretroviral therapy. J Acquir Immune Defic Syndr. 2002;31(2):253-255.

10. Carr A, Samaras K, Burton S, Law M, Freund J, Chisholm DJ, Cooper DA. A syndrome of peripheral lipodystrophy, hyperlipidaemia and insulin resistance in patients receiving HIV protease inhibitors. AIDS. 1998;12(7):F51-58.

11. Heath KV, Hogg RS, Chan KJ, Harris M, Montessori V, O’Shaughnessy MV, Montanera JS. Lipodystrophyassociated morphological, cholesterol and triglyceride abnormalities in a population-based HIV/AIDS treatment database. AIDS. 2001;15(2):231-239.

12. Rodriguez de la Concepcion ML, Domingo JC, Domingo P, Giralt M, Villarroya F. Uncoupling protein 1 gene 
expression implicates brown adipocytes in highly active antiretroviral therapy-associated lipomatosis. AIDS. 2004;18(6):959-960.

13. Guallar JP, Vila MR, Lopez-Gallardo E, Solano A, Domingo JC, Gamez J, Pineda M, et al. Altered expression of master regulatory genes of adipogenesis in lipomas from patients bearing tRNA(Lys) point mutations in mitochondrial DNA. Mol Genet Metab. 2006;89(3):283285.

14. Lo JC, Mulligan K, Noor MA, Schwarz JM, Halvorsen RA, Grunfeld C, Schambelan M. The effects of recombinant human growth hormone on body composition and glucose metabolism in HIV-infected patients with fat accumulation. J Clin Endocrinol Metab. 2001;86(8):34803487.

15. Gold J, Batterham M. Nandrolone decanoate; use in HIV-associated lipodystrophy syndrome: a pilot study. Int J STD AIDS. 1999;10(8):558.

16. Piliero PJ, Hubbard M, King J, Faragon JJ. Use of ultrasonography-assisted liposuction for the treatment of human immunodeficiency virus-associated enlargement of the dorsocervical fat pad. Clin Infect Dis. 2003;37(10):1374-1377.

17. Connolly N, Manders E, Riddler S. Suction-assisted lipectomy for lipodystrophy. AIDS Res Hum Retroviruses. 2004;20(8):813-815.

18. Shushan S, Cinamon U, Levy D, Sokolov M, Roth Y. Laryngeal cancer in acquired immunodeficiency syndrome. Int J STD AIDS. 2009;20(8):582-584.

19. National Comprehensive Cancer Network. Clinical practice guidelines in head and neck cancers. 2006. Available at www.nccn.org.

20. Bedimo RJ, McGinnis KA, Dunlap M, Rodriguez-Barradas MC, Justice AC. Incidence of non-AIDS-defining malignancies in HIV-infected versus noninfected patients in the HAART era: impact of immunosuppression. J Acquir Immune Defic Syndr. 2009;52(2):203-208.

21. Barry B, Gehanno P. [Squamous cell carcinoma of the ENT organs in the course of the HIV infection]. Ann Otolaryngol Chir Cervicofac. 1999;116(3):149-153.

22. Roland JT, Jr., Rothstein SG, Mittal KR, Perksy MS. Squamous cell carcinoma in HIV-positive patients under age 45. Laryngoscope. 1993;103(5):509-511.

23. 1993 revised classification system for HIV infection and expanded surveillance case definition for AIDS among adolescents and adults. MMWR Recomm Rep. 1992;41(RR-17):1-19.
24. National Comprehensive Cancer N, Forastiere AA, Ang KK, Brizel D, Brockstein BE, Burtness BA, Cmelak AJ, et al. Head and neck cancers. J Natl Compr Canc Netw. 2008;6(7):646-695.

25. Shimomura I, Funahashi T, Takahashi M, Maeda K, Kotani K, Nakamura T, Yamashita S, et al. Enhanced expression of PAI-1 in visceral fat: possible contributor to vascular disease in obesity. Nat Med. 1996;2(7):800803.

26. Mantzoros CS. The role of leptin in human obesity and disease: a review of current evidence. Ann Intern Med. 1999;130(8):671-680.

27. Tsao TS, Lodish HF, Fruebis J. ACRP30, a new hormone controlling fat and glucose metabolism. Eur J Pharmacol. 2002;440(2-3):213-221.

28. Sutinen J, Korsheninnikova E, Funahashi T, Matsuzawa Y, Nyman T, Yki-Jarvinen H. Circulating concentration of adiponectin and its expression in subcutaneous adipose tissue in patients with highly active antiretroviral therapy-associated lipodystrophy. J Clin Endocrinol Metab. 2003;88(4):1907-1910.

29. Watkins EB, Findlay P, Gelmann E, Lane HC, Zabell A. Enhanced mucosal reactions in AIDS patients receiving oropharyngeal irradiation. Int J Radiat Oncol Biol Phys. 1987;13(9):1403-1408.

30. Harris MA, Wise MS, Bonington A, Slevin NJ. Case report: radical radiotherapy for early laryngeal cancer in a patient with human immunodeficiency virus: no evidence of increased toxicity. Br J Radiol. 2004;77(918):519520 .

31. Kao GD, Devine P, Mirza N. Oral cavity and oropharyngeal tumors in human immunodeficiency virus-positive patients: acute response to radiation therapy. Arch Otolaryngol Head Neck Surg. 1999;125(8):873-876.

32. Bussu F, Micciche F, Rigante M, Dinapoli N, Parrilla C, Bonomo P, Cadoni G, et al. Oncologic outcomes in advanced laryngeal squamous cell carcinomas treated with different modalities in a single institution: A retrospective analysis of 65 cases. Head Neck. 2011.

33. Guallar JP, Gallego-Escuredo JM, Domingo JC, Alegre M, Fontdevila J, Martinez E, Hammond EL, et al. Differential gene expression indicates that 'buffalo hump' is a distinct adipose tissue disturbance in HIV-1-associated lipodystrophy. AIDS. 2008;22(5):575-584.

34. European AIDS Clinical Society (EACS) 2011 Guidelines (Version 6, October 2011). downloadable from www.europeanaidsclinicalsociety.org. 